Testing the GRACE follow-on triple mirror assembly

This content has been downloaded from IOPscience. Please scroll down to see the full text. 2014 Class. Quantum Grav. 31195004

(http://iopscience.iop.org/0264-9381/31/19/195004)

View the table of contents for this issue, or go to the journal homepage for more

Download details:

IP Address: 194.94.224.254

This content was downloaded on 15/12/2014 at 09:31

Please note that terms and conditions apply. 


\title{
Testing the GRACE follow-on triple mirror assembly
}

\author{
R Fleddermann ${ }^{1}, \mathbf{R} \mathbf{L}$ Ward $^{1}, \mathbf{M}$ Elliot ${ }^{1}, \mathbf{D}$ M Wuchenich ${ }^{1}$, \\ F Gilles ${ }^{2}, \mathbf{M ~ H e r d i n g ~}^{2}$, K Nicklaus ${ }^{2}, \mathbf{J ~ B r o w n ~}^{3}, \mathbf{J ~ B u r k e}^{4}$, \\ S Dligatch $^{3}$, D I Farrant ${ }^{3}, K$ L Green ${ }^{3}$, J A Seckold ${ }^{3}$, \\ M Blundell $^{5}$, R Brister ${ }^{5}$, C Smith ${ }^{5}$, B S Sheard ${ }^{6}$, G Heinzel ${ }^{6}$, \\ K Danzmann ${ }^{6}$, B Klipstein ${ }^{7}$, D E McClelland ${ }^{1}$ and \\ D A Shaddock ${ }^{1}$
}

\begin{abstract}
${ }^{1}$ Australian National University, Department of Quantum Science, 2 Science Rd, Acton, ACT 2000, Australia

${ }^{2}$ SpaceTech GmbH Immenstaad, Seelbachstr. 13, D-88090 Immenstaad, Germany

${ }^{3}$ Australian Centre for Precision Optics, CSIRO Materials Science and Engineering, PO Box 218, Lindfield, 2070 NSW, Australia

${ }^{4}$ BIAS-Bremer Institut für angewandte Strahltechnik gGmbH, Abteilung Optische Messtechnik, Klagenfurter Str. 2, D-28359 Bremen, Germany

${ }^{5}$ Electro Optic Systems Pty Limited, EOS House, Mount Stromlo Observatory, Cotter Road, Weston Creek ACT 2611, Australia

${ }^{6}$ Max Planck Institute for Gravitational Physics (Albert Einstein Institute) and Institute for Gravitational Physics of the Leibniz Universität Hannover, Callinstraße 38, D-30167 Hannover, Germany

${ }^{7}$ Jet Propulsion Laboratory, California Institute of Technology, Pasadena, CA 91 109, USA
\end{abstract}

E-mail: Roland.Fleddermann@anu.edu.au

Received 21 February 2014, revised 22 June 2014

Accepted for publication 23 July 2014

Published 16 September 2014

\begin{abstract}
We report on the successful testing of the GRACE follow-on triple mirror assembly (TMA) prototype. This component serves to route the laser beam in a proposed follow-on mission to the Gravity Recovery and Climate Explorer (GRACE) mission, containing an optical instrument for space-based distance measurement between satellites. As part of this, the TMA has to meet a set of stringent requirements on both the optical and mechanical properties. The purpose of the TMA prototype testing is to establish the feasibility of the design, materials choice and fabrication techniques. Here we report on co-alignment testing of this device to the arc second ( $5 \mu \mathrm{rad})$ level and thermal alignment stability testing to $1 \mu \mathrm{rad} \mathrm{K}^{-1}$.
\end{abstract}


Keywords: gravity recovery and climate explorer, GRACE, triple mirror assembly, laser ranging instrument, GRACE follow-on, retroreflectors, coalignment measurements

PACS numbers: 07.60.Ly, 93.85.Hj, 42.79.Fm, 07.87.+v, 81.70.Bt, 93.85.+q

(Some figures may appear in colour only in the online journal)

\section{Introduction}

The triple mirror assembly (TMA) will be an integral part of the Gravity Recovery and Climate Explorer (GRACE) [1, 2] follow-on mission's laser ranging instrument (LRI). The purpose of the LRI is to demonstrate laser interferometric ranging in space between independent satellites for the first time. If successful, this has the potential to improve upon the accuracy of the measurement of Earth's gravitational field from space by providing a more accurate measurement of the distance between the two GRACE satellites.

The LRI is a technology demonstrator, and as such, must not interfere with the existing instrumentation on board the GRACE satellites. While it would be desirable to measure the distance between the two satellites along their line of sight to avoid coupling satellite rotations into the distance measurement, this will not be possible, due to the existing $\mathrm{K}_{\mathrm{a}}$-band microwave interferometric distance measurement system and cold-gas tanks occupying this region. Therefore, the laser beams must be routed around the line of sight between the two spacecraft. This is to be accomplished using a lateral transfer hollow retro-reflector, called the TMA in the context of this project.

This TMA acts like a corner-cube and removes coupling of pointing variations in the distance measurement. By mounting only the parts of the cube that are required for beam routing on a very rigid tube, as shown in figure 1 , it saves on both size and mass over a conventional design. Detailed information on the fabrication process of this device can be found in [3].

As discussed there, this TMA design uses carbon fiber reinforced polymer (CFRP) as a structural material. While this material has been used in space applications extensively (see examples in [4]) and accurate tests on the optical stability have been performed $([5,6])$, results found in these studies do not directly translate to the TMA because the properties of CFRP strongly depend on the fabrication process. Furthermore, the effects of potentially nonuniform thermal expansion, which is commonly observed in CFRP, on the alignment of the optics cannot be modeled in a straightforward way. Therefore, direct tests of the critical performance parameters of this prototype are still required.

The TMA has to fulfill a number of stringent requirements in order to be useful for the GRACE follow-on mission. For the measurement scheme to succeed, the incoming and outgoing beams on each spacecraft must be co-aligned to within $20 \mu \mathrm{rad}$ under all spacecraft operating conditions. For space-qualification it is also necessary for the device to adhere to the European Cooperation for Space Standardization test standards described in [7]. Among other things, these standards require vibration tests to ensure that the device can survive the launch on a rocket. We also conducted tests to ensure that the co-alignment requirement is met over the predicted operational temperature range of $20 \pm 10^{\circ} \mathrm{C}$.

An overview of the requirements to be met by the TMA and the tests performed to verify them can be found in table 1 . There is also a requirement on the length stability of the device. However, the tests performed to verify the length stability require a completely different setup. We chose to implement one that resembles the measurement architecture on board the GRACE follow-on space craft much more closely. The length stability results are therefore 


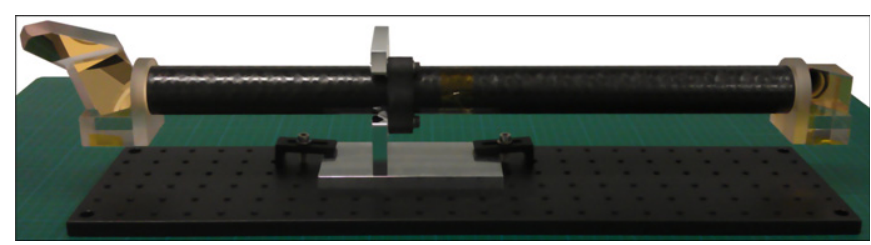

Figure 1. Photograph of the triple mirror assembly on an optical breadboard.

Table 1. List of requirements to be met and tests performed.

\begin{tabular}{lcc}
\hline Description & Requirement & Reference \\
\hline Length stability & $<25 \mathrm{~nm} / \sqrt{\mathrm{Hz}}(2 \mathrm{mHz}-0.1 \mathrm{~Hz})$ & {$[8]$} \\
Initial co-alignment & $\pm 20 \mu \mathrm{rad}$ & Section 2.1 \\
Thermal co-alignment & $<1 \mu \mathrm{rad} \mathrm{K}^{-1}$ & Section 2.2 \\
Vibration & Survival + no resonances $<100 \mathrm{~Hz}$ & section 2.3 \\
\hline
\end{tabular}

presented separately along with the first demonstration of the race-track interferometry architecture [8].

Here, we report on tests performed on a prototype model of the TMA. The prototype serves to tests the manufacturing and design approach before actual engineering models and flight models are built. It is built to the same specifications. Tests were conducted to verify the relevant performance requirements, but cleanliness and quality management requirements are not an issue as they have no effect on the measurements we conducted.

\section{Tests performed}

\subsection{Initial co-alignment}

2.1.1. Requirement. The parallelism of the incoming and outgoing beam is a key requirement of the TMA. It influences how much (if any) of the light that is returned from one spacecraft can be detected by the other spacecraft. The deviation from perfect antiparallelism of the incoming and outgoing beam has to be kept below $20 \mu \mathrm{rad}$ [9].

2.1.2. Test setup. The characterization of corner cubes at the arc-second level is most commonly performed by using a Fizeau interferometer using a setup as discussed in e.g. [10]. However, here this technique could not be used, because it requires the interferometer to have an aperture of at least the distance between incoming and outgoing beam, which in this case is $600 \mathrm{~mm}$. Due to the fact that the aperture of the interferometer available to us was only $100 \mathrm{~mm}$, we used a variation of the conventional Fizeau interferometer measurement setup.

Figure 2 shows a schematic of this measurement setup. Here, the light from the polarization-based common path simultaneous phase-shifting Fizeau interferometer was first passed through a reference bar, polished to make one of the surfaces almost perfectly flat, so it can serve as a reference in this measurement (supplied by CSIRO). The reference bar's flatness was measured independently by CSIRO. Analysis of the measured profiles indicated that the maximum error in a measurement with a $20 \mathrm{~mm}$ aperture (as was the case in this setup) was $3.25 \mu \mathrm{rad}$ in the direction across the bar (vertical) and $5.80 \mu \mathrm{rad}$ in the direction along the bar (horizontal) [11]. 
reference bar

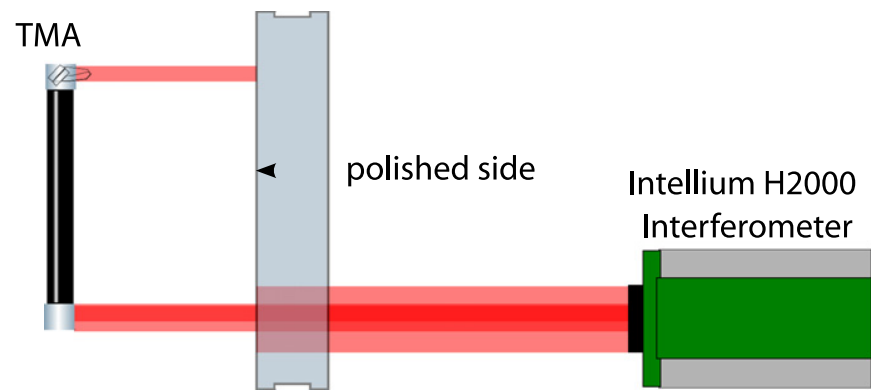

Figure 2. Test setup used to measure the static co-alignment of the incoming and outgoing beams to/from the TMA.
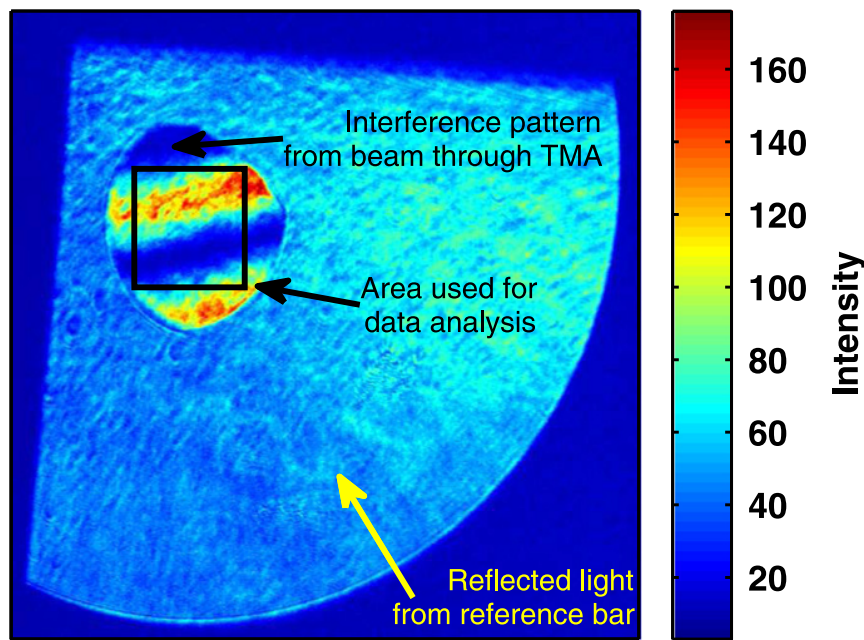

Figure 3. Typical image from co-alignment test showing the detected light intensity in false color. The black box highlights the area used for data analysis as detailed in figure 4.

Part of the beam reflected off the left surface of this reference bar served as a reference beam. The remaining light passed through the reference bar and was reflected onto the other end of the reference bar by the TMA. A fraction of the light was reflected by this surface of the reference bar again, traveled back through the TMA before interfering with the reference beam. The resulting interference pattern was then recorded by the camera inside the interferometer. The visibility of this interference pattern was typically about 90 per cent as the reflectivity of the gold-coated TMA mirrors for the wavelength used in the test $(634.3 \mathrm{~nm})$ is very high.

However, using this setup one can only obtain a static interference image as opposed to the optical path length difference that is normally generated automatically by the interferometer's software. For this mode of operation, the interferometer requires a relative angle between the reference beam and the measurement beam, which is not achievable here. 


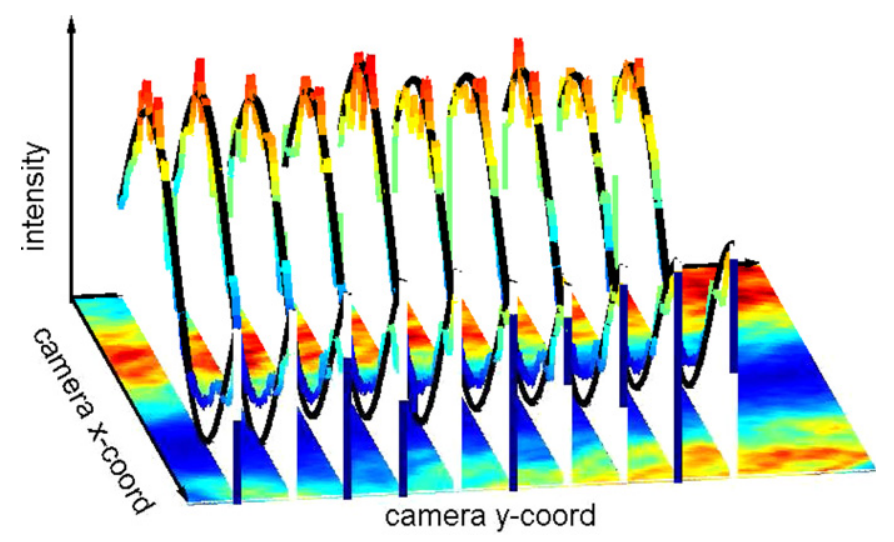

Figure 4. Illustration of the data analysis strategy used to measure the static misalignment between the incoming and outgoing beams using interferograms obtained in a non-phase shifting interferometry configuration. The interference image is depicted in false color representation on the $x$ - $y$-plane, sine-waves fitted to cross-sections in the $x$-direction are shown in black together with the color coded data at these positions, both in $3 \mathrm{D}$ representation.

This means that the reference beam can only interfere with itself and not with the measurement beam, which is ordinarily phase shifted with respect to the reference beam.

Figure 3 shows an example of an interference image obtained using our measurement setup. The detected intensity inside the interferometer's aperture is shown in false color, with red indicating a high intensity and blue hues indicating low intensity. One can see the low intensity reflected light from the fraction of the reference bar that is inside the aperture in the background, with a roughly spherical area in the upper left hand corner showing the interference fringes from light that passed through the TMA's spherical aperture. A black square highlights the fraction of the image on which image analysis was performed using MATLAB ${ }^{\odot}$, to calculate the relative angle between the incoming and outgoing beams.

The data analysis is illustrated in more detail in figure 4. Here, only the area highlighted by the black rectangle in figure 3 is shown in the same false color depiction. On top of the square, sine waves that were fitted to slices through the interferogram are shown, indicating where this process was performed and how the sine waves fit the data. The nonlinear fit used for this yielded both phase and frequency information, which was then used to calculate the relative angle between the beam that has passed through the TMA twice and the reference beam that was reflected by the reference bar directly. Assuming that the reference bar is flat and does not introduce an additional angle, this yields twice the angular error of the TMA.

2.1.3. Results. To minimize the error that is introduced by the data analysis technique and by refractive index variations of the air in the lab due to air currents, this measurement was repeated 100 times, with each measurement generating three phase shifted versions of the interference image. Data from all these measurements was used to calculate the deviation from perfect alignment in the horizontal and vertical direction separately. Figure 5 shows a plot of the angles found in each measurement along with the average angles. Red circles indicate the 1-, 2- and 3-sigma standard deviations. The average horizontal angular deviation was found to be $3.28 \mu \mathrm{rad}$ while the average vertical deviation was $11.59 \mu \mathrm{rad}$. This results in 


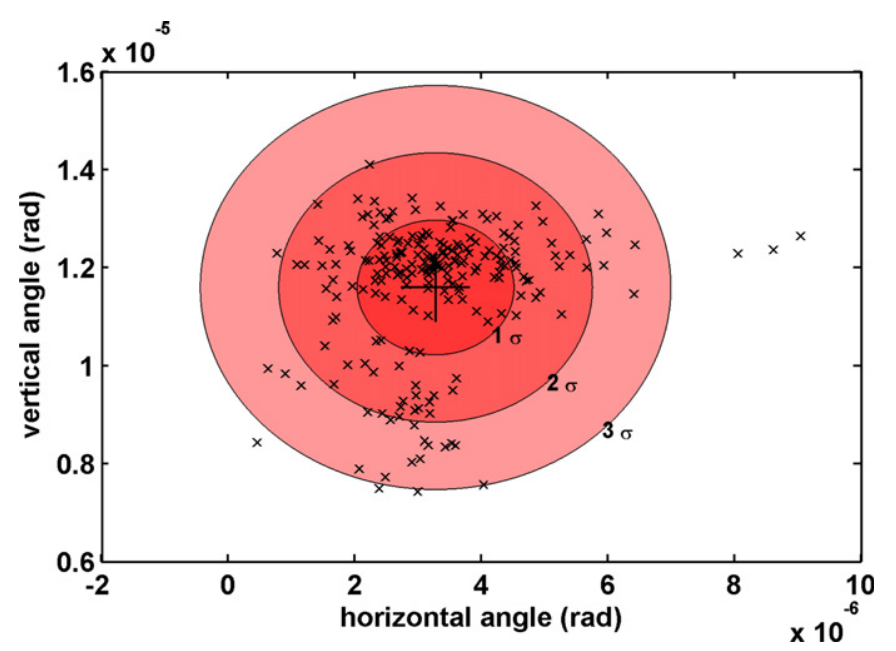

Figure 5. Results of the static co-alignment measurement, showing a horizontal angle of $3.28 \mu \mathrm{rad}$ and a vertical angle of $11.59 \mu \mathrm{rad}$, resulting in a magnitude of the angle of $12.05 \mu \mathrm{rad}$.

a combined magnitude of the angular deviation from perfect 180 degrees of $12.05 \mu \mathrm{rad}$, comfortably meeting the $20 \mu \mathrm{rad}$ requirement.

\subsection{Thermal co-alignment}

2.2.1. Requirement. For the TMA to operate correctly in the GRACE follow-on mission, it has to be ensured that the co-alignment of the beams does not change significantly over the operating temperature range of $20^{\circ} \mathrm{C} \pm 10^{\circ} \mathrm{C}$, because the temperature on board the spacecraft can only be predicted to within this margin. It is therefore essential that the TMA's alignment does not change by more than an additional $20 \mu \mathrm{rad}$ over this temperature range, or-equivalently - that the thermal alignment coefficient is below $1 \mu \mathrm{rad} \mathrm{K}^{-1}$.

As it was not possible to test the requirement from $10{ }^{\circ} \mathrm{C}$ to $30^{\circ} \mathrm{C}$ because a thermal chamber that allows cooling and has optical access for measurement of the co-alignment was not available, it was decided to conduct the tests in a custom built thermal enclosure with optical access which only allows heating. This was justified under the reasonable assumption that the thermal expansion/deformation is linear, and therefore the relative alignment change should be identical at slightly lower temperatures, while allowing a much simpler construction of the test chamber.

2.2.2. Test setup. As discussed above, a special thermal enclosure was built for the verification of this requirement. It allowed the TMA's environmental temperature to be varied from room temperature to approximately $40^{\circ} \mathrm{C}$.

Figure 6 shows a schematic representation of the measurement setup used to measure the change of alignment at different temperatures. Due to the fact that it is not required to measure the absolute alignment between the incoming and outgoing beams, but only the change in alignment with change of environmental temperature, the interferometer's phase-shifting measurement capabilities were used to track the angle of the mirror surfaces with respect to a stable reference outside the thermal chamber. 


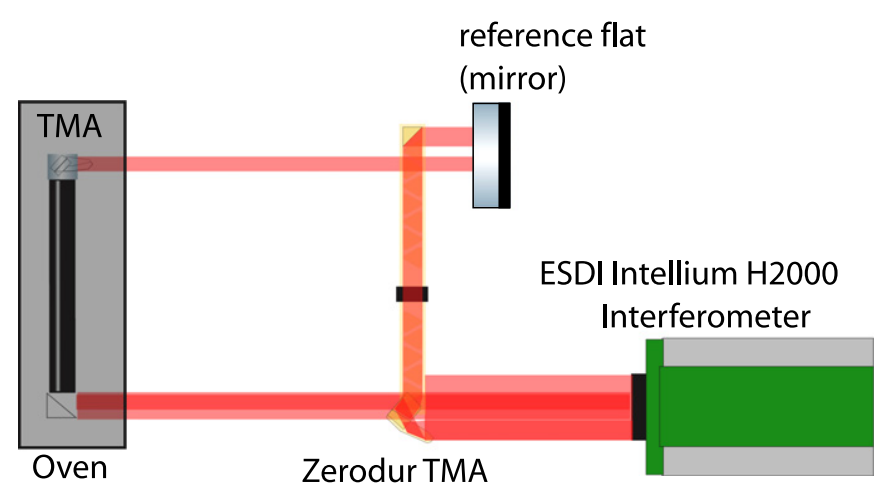

Figure 6. Schematic of the TMA thermal pointing stability test setup.

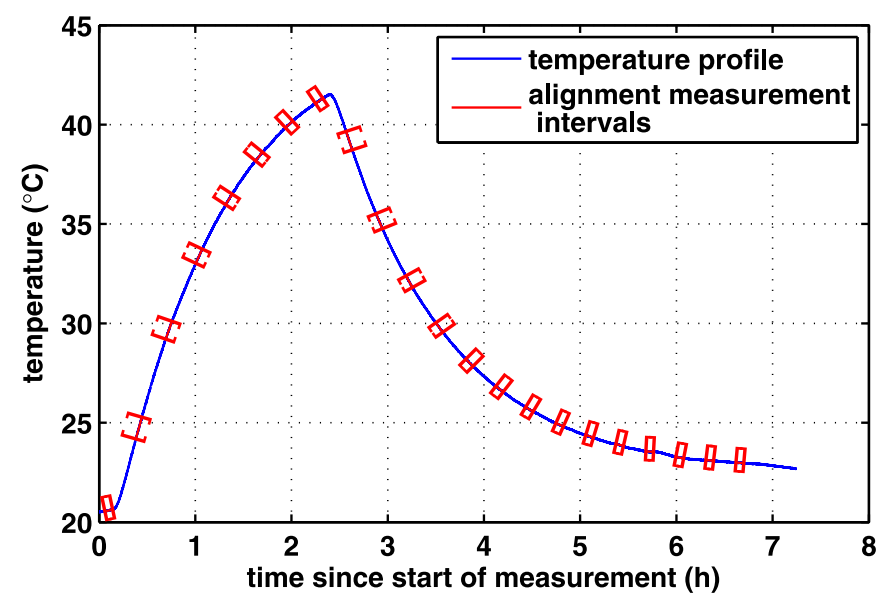

Figure 7. Temperature profile used in the thermal co-alignment tests.

A prototype of a TMA made of ULE and Zerodur ${ }^{\odot}$ was used as the stable reference. This device was expected to be negligibly affected by residual thermal radiation escaping from the oven shielding due to the ultra-low coefficient of thermal expansion of these materials. The relative angle between this reference and the CFRP TMA prototype in the oven was monitored at 10 minute intervals using automated measurements from the interferometer, while the oven was gradually heated to about $40{ }^{\circ} \mathrm{C}$ and then left to cool down in the room temperature environment, resulting in a temperature profile as depicted in figure 7 . A slow rise in temperature over the first approximately 2 hours was followed by an even slower decrease in temperature over the following 5 hours.

The temperature inside the oven was monitored using PT10k positive temperature coefficient (PTC) resistors, read out by a Pico Technology PT-104 Platinum Resistance Data Logger. As the relative change in resistance of platinum PTC sensors is very well known, this leads to a very accurate temperature measurement.

The measurement produced by the $\mathrm{H} 2000$ was in the form of an optical path difference (OPD). To convert this to a co-alignment measurement, the OPD for each region was decomposed into a set of Zernike polynomials [12]. Only the Zernike(1,1) and Zernike(-1,1), which represent horizontal and vertical tilts, were of interest. These two Zernike coefficients 


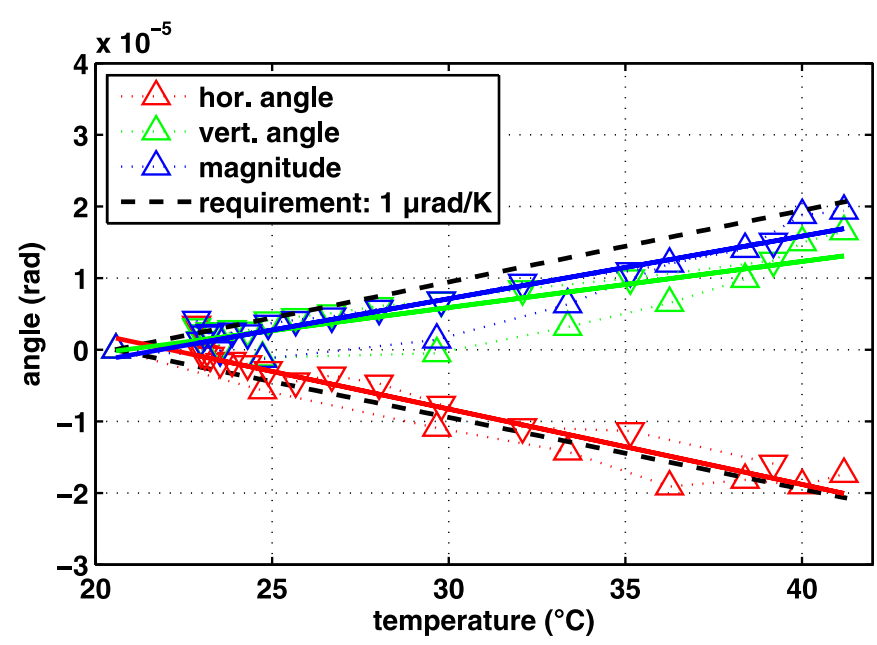

Figure 8. Results of thermal co-alignment measurements.

were used to determine the relative horizontal and vertical angles between the light that has transited the CFRP TMA and the light that has transited the reference. Any change in the angles' difference was attributed to the thermal deformation of the CFRP TMA.

2.2.3. Results. Figure 8 shows the change in both horizontal and vertical beam alignment as well the change in magnitude of the deviation from the static pointing determined in the previous measurements, plotted versus the temperature inside the oven. As one can see, the vertical angle, represented by the green triangles, increased with an increase in temperature, while the horizontal angle, represented by the red triangles, decreased. The temperature coefficient of the magnitude, depicted in blue, is dominated by the change in the vertical direction due to the relatively large static misalignment in this direction. One can also notice a slight hysteresis upon close inspection of the part of the traces observed during an increase of the temperature (upwards pointing triangles, solid line) and the part measured during the following decrease in temperature (downwards pointing triangles, dashed line), which was most pronounced in the vertical angle.

Note, that while the plot shows only the difference from the initial angle measurement, the static misalignment found in the previous section was added to the data before calculating the magnitude. This allows for a realistic assessment of the magnitude of the angular error. It is required that the change in magnitude of the misalignment be less than $20 \mu \mathrm{rad}$ over the whole range or, equivalently, that the thermal alignment coefficient is less than $1 \mu \mathrm{rad} \mathrm{K}^{-1}$.

Comparison with the requirement of less than $1 \mu \mathrm{rad} \mathrm{K}^{-1}$ represented by the black dashed lines in figure 8 shows that the TMA prototype meets this requirement, although compliance is marginal when looking at the change in horizontal angle in isolation. However, for the received power to remain above the detection threshold the magnitude of the change in angle is the relevant number. This value has been found to meet this requirement in this test.

\subsection{Vibration testing}

2.3.1. Requirement. Vibration testing of the TMA is required to ensure that the device can survive a rocket launch. Such tests serve to identify any mechanical resonances in the assembly and to confirm that the device is strong enough to survive high levels of 


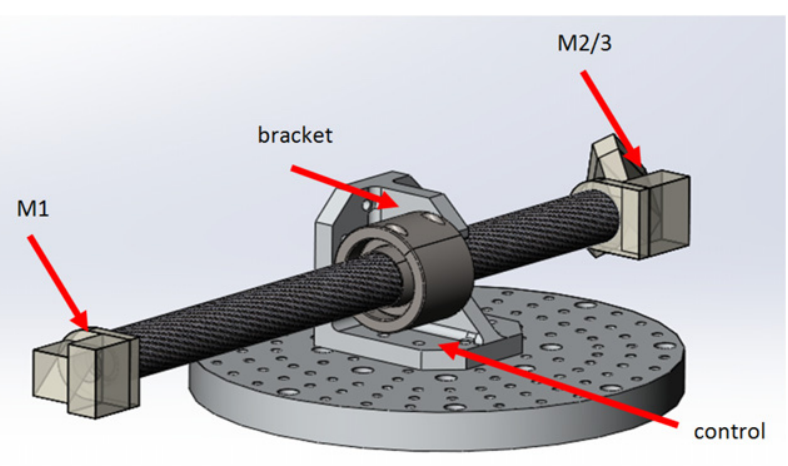

Figure 9. Test configuration for the spacecraft $x$-axis vibration tests. Red arrows indicate the positions where accelerometers were installed to measure the actual acceleration levels achieved during the test.

Table 2. Input acceleration levels for the high level swept-sine test.

\begin{tabular}{lc}
\hline Frequency range in $\mathrm{Hz}$ & Input \\
\hline $5-20$ & $11 \mathrm{~mm}$ \\
$20-60$ & $20 \mathrm{~g}$ \\
$60-100$ & $6 \mathrm{~g}$ \\
\hline
\end{tabular}

Table 3. Input acceleration levels for the random vibration test. These values result in an acceleration level of $10.7 \mathrm{~g}$ rms.

\begin{tabular}{lc}
\hline Frequency range in $\mathrm{Hz}$ & Input \\
\hline $20-100$ & $+3 \mathrm{~dB} / \mathrm{oct}$ \\
$100-300$ & $\mathrm{PSD}=0.2 \mathrm{~g}^{2} / \mathrm{Hz}$ \\
$300-2000$ & $-5 \mathrm{~dB} / \mathrm{oct}$ \\
\hline
\end{tabular}

acceleration. Testing was done according to the European Space Agency (ESA) standards for space qualification tests [7].

These standards require two types of tests to be performed. In the first type, a sinusoidal acceleration serves to test for workmanship errors. In the second test, random vibration emulates the worst case acceleration environment encountered on the launch spacecraft. The respective input acceleration levels for spacecraft components from the standards adopted for these tests are listed in tables 2 and 3.

2.3.2. Test setup. Vibration testing was performed using a Brüel \& Kjær LDS V830 vertical shaker table. The TMA was mounted to its interface plate using a specially designed mounting bracket with no mechanical resonance frequencies below $2 \mathrm{kHz}$. The shaker table was then programmed to perform a series of different tests as described in more detail below. Acceleration levels were monitored in three different positions by three-axis accelerometers from PCB Piezotronics of type J353B33. The positions in which these were attached are 

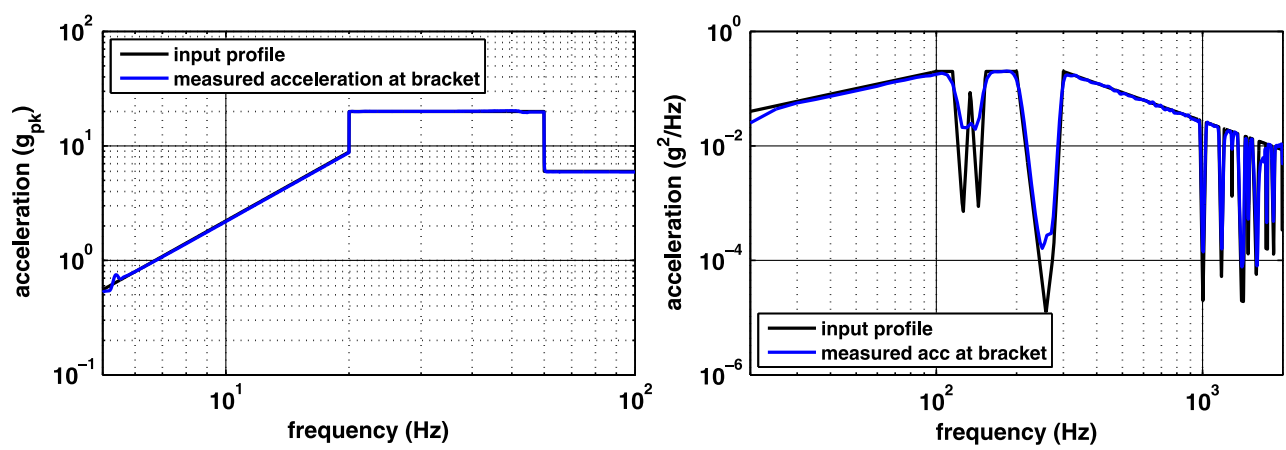

Figure 10. Input spectra for vibration tests. Left: high level swept-sine test, right: random vibration test. Black traces represent programmed acceleration levels, blue traces show measured acceleration levels.

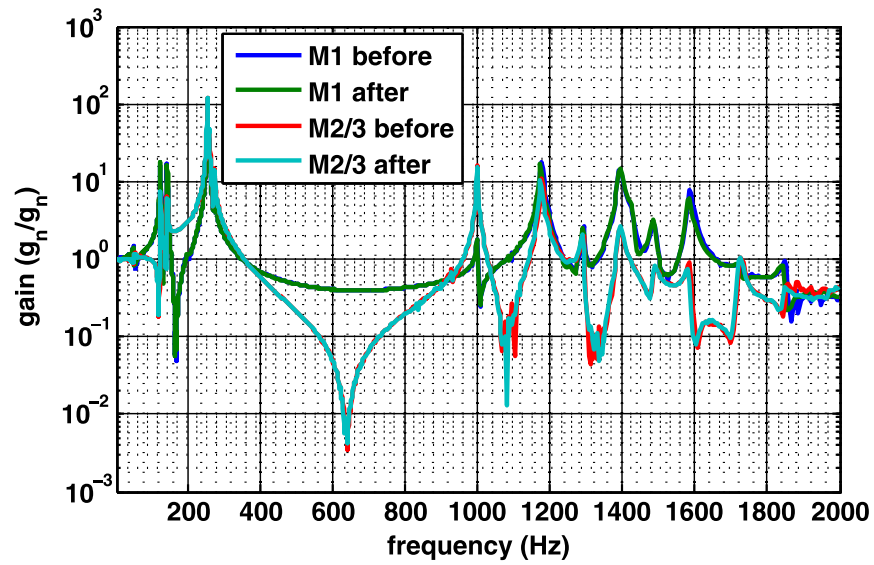

Figure 11. Comparison of swept-sine resonance search results before and after high level vibration testing.

highlighted by red arrows in figure 9, which depicts a CAD rendering of the TMA and the mounting bracket. As also indicated in this figure, a fourth single-axis accelerometer was installed at the bottom of the TMA mounting bracket. This sensor was used for closed-loop control of the shaker table's input acceleration.

The test procedure consisted of an initial swept-sine resonance search to identify strong resonances which require notching to avoid over-testing in later tests, followed by a highlevel swept-sine test to verify that the prototype can withstand accelerations of up to $20 \mathrm{~g}_{\mathrm{rms}}$. The input acceleration levels in the frequency range of the test are listed in table 2. The sweep rate was set to 2 octaves per minute, resulting in a test duration of a little over 2 minutes. Figure 10 shows the input accelerations for this test on the left-hand side. The acceleration level increases linearly between 5 and $20 \mathrm{~Hz}$, then it jumps to $20 \mathrm{~g}$ for frequencies between 20 and $60 \mathrm{~Hz}$ and goes back down to $6 \mathrm{~g}$ between 60 and $100 \mathrm{~Hz}$.

After this, the swept-sine resonance test was repeated to identify potential changes in the resonance frequencies, indicating possible internal damage of the material not discernible in a visual inspection of the test item. Finally, a random vibration test was performed, simulating a worst case launch environment in accordance with the input levels given in table 3 . 
Table 4. Resonance frequencies and observed during the swept-sine vibration tests.

\begin{tabular}{lcc}
\hline Mode & $\begin{array}{c}\text { Measured resonance } \\
\left(\mathrm{H}_{z}\right)\end{array}$ & $\begin{array}{c}\text { Amplitude } \\
(\mathrm{g} / \mathrm{g})\end{array}$ \\
\hline 1 & 126 & 18 \\
2 & 145 & 16 \\
3 & 256 & 118 \\
4 & 275 & 14 \\
5 & 1002 & 15 \\
\hline
\end{tabular}

Additionally, notches were inserted into the excitation spectrum of this test to limit the acceleration observed at the mirror interfaces to $100 \mathrm{~g}(3 \sigma)$, resulting in the input acceleration spectral density depicted in the right-hand side of figure 10. The input level noise spectral density is represented by the black trace in the figure, which ramped up between 20 and $100 \mathrm{~Hz}$ to a level of $0.2 \mathrm{~g}^{2} / \mathrm{Hz}$, remained flat between 100 and $300 \mathrm{~Hz}$ and decreased again for even higher frequencies. Additionally, one can see a number of frequency ranges where the input acceleration is strongly attenuated due to the notches that were inserted at the resonance frequencies of the TMA. These notches led to a reduction of the expected acceleration level of $10.7 \mathrm{~g}_{\text {rms }}$ down to $9.16 \mathrm{~g}_{\mathrm{rms}}$. The actual measured input acceleration level at the sensor used for feedback control of the input acceleration was $9.02 \mathrm{~g}_{\text {rms }}$, while the measured acceleration at either mirror end was $9.49 \mathrm{~g}_{\mathrm{rms}}$ at the M1 end and $11.5 \mathrm{~g}_{\mathrm{rms}}$ at the M2/3 end.

After the TMA had been subjected to this test, the swept-sine resonance search was repeated a second time to ensure that resonance frequencies still had not shifted and a visual inspection was carried out.

2.3.3. Results. Figure 11 shows a comparison of the pre-test and post-test resonance spectra found in the low-level swept-sine tests. The resonance frequencies have not changed, indicating that no internal structural damage occurred during the testing. The minor differences in the frequency range between 1800 and $2000 \mathrm{~Hz}$ are consistent with minimal changes from run to run and do not indicate any structural problems. A final visual inspection also revealed no visible material defects.

Table 4 lists the resonance frequencies found during the first swept-sine tests and the respective amplitudes. As one can see, the first resonance was observed at $126 \mathrm{~Hz}$, which is consistent with the requirement that there shall be no resonances below $100 \mathrm{~Hz}$. This indicates that the prototype is stiff enough to meet the requirements, and serves as a guideline for the design of an engineering model. The amplitude values found here were used to set the depth of the notches for the random vibration test.

\section{Conclusion}

We presented the results of space qualification and testing of a prototype of the GRACE follow-on mission's TMA, a device required to route the beam around existing hardware onboard the spacecraft. We confirmed that the initial co-alignment of the incoming and outgoing beam meets the requirement of $20 \mu \mathrm{rad}$, using a technique adapted for this specialized application and a custom made reference flat manufactured solely for this purpose.

Furthermore, we were able to measure the change in co-alignment over a range of temperatures spanning $20^{\circ} \mathrm{C}$. The measurements showed that this change is consistent with 
the requirement of less than $1 \mu \operatorname{rad}$ per ${ }^{\circ} \mathrm{C}$, using a measurement technique involving a second prototype.

Finally we confirmed that the apparatus is strong enough to be able to withstand the vibrations occurring during a launch into space on a rocket, as described in section 2.3 . The random and sinusoidal vibration tests did not reveal structural damage to the device, nor were any changes in the resonance frequencies of the device observed.

In summary, we showed that the current prototype of the TMA, as built and designed by CSIRO, Electro-Optic Systems, SpaceTech GmbH Immenstaad and the ANU, meets all the requirements imposed by the GRACE follow-on mission. This demonstrates that it is feasible to use such a retro-reflecting device with very strict alignment requirements in a space mission, addressing one of the major technology challenges in the development of the LRI demonstrator for the mission.

\section{Acknowledgements}

The TMA development was funded by the Australian Space Research Program, an initiative of the Australian Government, with contributions from the German Federal Ministry of Education and Research, the German Aerospace Centre, Australia's Commonwealth Scientific and Industrial Research Organisation, and the Deutsche Forschungsgemeinschaft (DFG) within the Cluster of Excellence QUEST (Centre for Quantum Engineering and Space-Time Research).

\section{References}

[1] Tapley B D, Bettadpur S, Watkins M and Reigber C 2004 The gravity recovery and climate experiment: mission overview and early results Geophys. Res. Lett. 31 L09607

[2] Wiese D N, Nerem R S and Lemoine F G 2011 Design considerations for a dedicated gravity recovery satellite mission consisting of two pairs of satellites J. Geod. 86 81-98

[3] Ward R L et al 2014 The design and construction of a prototype lateral-transfer retro-reflector for inter-satellite laser ranging Class. Quantum Grav. 31095015

[4] Rawal S P and Goodman J W 2001 Space applications ASM Handbook (Materials Park, OH: ASM International) vol 21, pp 1033-42

[5] Sanjuán J, Preston A, Korytov D, Spector A, Freise A, Dixon G, Livas J and Mueller G 2011 Carbon fiber reinforced polymer dimensional stability investigations for use on the laser interferometer space antenna mission telescope Rev. Sci. Instrum. 82124501

[6] Edeson R, Morris N, Tatnall A and Aglietti G S 2009 Dimensional stability testing on a space optical bench structure AIAA J. 47 219-28

[7] European Cooperation for Space Standardization 2012 Space engineering: testing www.ecss.nl/ forums/ecss/dispatch.cgi/standards/showFile/100824/d20120604160035/No/ECSS-E-ST-1003C(1June2012).pdf

[8] Wuchenich D M R 2014 Inter-satellite laser interferometry PhD Thesis Australian National University

[9] Sheard B S, Heinzel G, Danzmann K, Shaddock D A, Klipstein W M and Folkner W M 2012 Intersatellite laser ranging instrument for the GRACE follow-on mission J. Geod. 86 1083-95

[10] Mantravadi M V and Malacara D 2007 Optical Shop Testing 3rd edn (New York: Wiley)

[11] Schütze D, Farrant D, Shaddock D A, Sheard B S, Heinzel G and Danzmann K 2014 Measuring coalignment of retroreflectors with large lateral incoming-outgoing beam offset Rev. Sci. Instrum. 85035103

[12] Wang J Y and Silva D E 1980 Wave-front interpretation with Zernike polynomials Appl. Opt. 19 $1510-8$ 